\title{
Clinical phenotypes in adult patients with bronchiectasis
}

\author{
Stefano Aliberti ${ }^{1}$, Sara Lonni ${ }^{1}$, Simone Dore ${ }^{2}$, Melissa J. McDonnell ${ }^{3}$, \\ Pieter C. Goeminne ${ }^{4,5}$, Katerina Dimakou ${ }^{6}$, Thomas C. Fardon ${ }^{7}$, \\ Robert Rutherford ${ }^{3}$, Alberto Pesci ${ }^{1}$, Marcos I. Restrepo ${ }^{8}$, Giovanni Sotgiu ${ }^{2}$ and \\ James D. Chalmers ${ }^{7}$
}

Affiliations: 'School of Medicine and Surgery, University of Milan Bicocca, AO San Gerardo, Monza, Italy. ${ }^{2}$ Clinical Epidemiology and Medical Statistics Unit, Dept of Biomedical Sciences, University of Sassari Research, Medical Education and Professional Development Unit, AOU Sassari, Sassari, Italy. ${ }^{3}$ Dept of Respiratory Medicine, Galway University Hospitals, Galway, Ireland. ‘Dept of Respiratory Medicine, University Hospital Gasthuisberg, Leuven, Belgium. ${ }^{5}$ Dept of Respiratory Medicine, UZ Leuven, Leuven, Belgium. ${ }^{6} 5$ th Pulmonary Dept, "Sotiria" Chest Hospital, Athens, Greece. ${ }^{7}$ Tayside Respiratory Research Group, University of Dundee, Dundee, UK. ${ }^{8}$ Division of Pulmonary Diseases and Critical Care, South Texas Veterans Health Care System and University of Texas Health Science Center, San Antonio, TX, USA.

Correspondence: Stefano Aliberti, School of Medicine and Surgery, University of Milan Bicocca, AO San Gerardo, Via Pergolesi 33, 20052 Monza, Italy. E-mail: stefano.alibertiđunimib.it

ABSTRACT Bronchiectasis is a heterogeneous disease. This study aimed at identifying discrete groups of patients with different clinical and biological characteristics and long-term outcomes.

This was a secondary analysis of five European databases of prospectively enrolled adult outpatients with bronchiectasis. Principal component and cluster analyses were performed using demographics, comorbidities, and clinical, radiological, functional and microbiological variables collected during the stable state. Exacerbations, hospitalisations and mortality during a 3-year follow-up were recorded. Clusters were externally validated in an independent cohort of patients with bronchiectasis, also investigating inflammatory markers in sputum.

Among 1145 patients (median age 66 years; 40\% male), four clusters were identified driven by the presence of chronic infection with Pseudomonas aeruginosa or other pathogens and daily sputum: "Pseudomonas" (16\%), "Other chronic infection" (24\%), "Daily sputum" (33\%) and "Dry bronchiectasis" (27\%). Patients in the four clusters showed significant differences in terms of quality of life, exacerbations, hospitalisations and mortality during follow-up. In the validation cohort, free neutrophil elastase activity, myeloperoxidase activity and interleukin- $1 \beta$ levels in sputum were significantly different among the clusters.

Identification of four clinical phenotypes in bronchiectasis could favour focused treatments in future interventional studies designed to alter the natural history of the disease.

@ERSpublications

Daily sputum and chronic infection with Pseudomonas or other bacteria define clinical phenotypes in bronchiectasis http://ow.ly/W4H9m

For editorial comment see Eur Respir J 2016; 47: 1037-1039 [DOI: 10.1183/13993003.00163-2016].

This article has supplementary material available from erj.ersjournals.com

Received: Oct 052015 | Accepted after revision: Dec 082015 | First published online: Feb 042016

Support statement: This study was supported by the European Multicentre Bronchiectasis Audit and Research Collaboration (EMBARC; www.bronchiectasis.eu). EMBARC is a European Respiratory Society Clinical Research Collaboration and has received funding from the European Respiratory Society, Bayer HealthCare and Aradigm Corporation. J.D. Chalmers acknowledges fellowship support from the Medical Research Council and the Wellcome Trust. M.J. McDonnell acknowledges fellowship support from the European Respiratory Society/European Lung Foundation and Health Research Board, Ireland. M.I. Restrepo's time is partially protected by Award Number K23HL096054 from the National Heart, Lung and Blood Institute. The content is solely the responsibility of the authors and does not necessarily represent the official views of the National Heart, Lung and Blood Institute or the National Institutes of Health. The funding agencies had no role in the preparation, review or approval of the manuscript. The views expressed in this article are those of the authors and do not necessarily represent the views of the Department of Veterans Affairs.

Conflict of interest: Disclosures can be found alongside the online version of this article at erj.ersjournals.com

The content of this work is not subject to copyright. Design and branding are copyright @ERS 2016. 


\section{Introduction}

Bronchiectasis is a chronic airway disease characterised by irreversibly damaged and dilated bronchi leading to recurrent episodes of bronchial sepsis. This results in poor mucus clearance, and a vicious cycle of persistent bacterial colonisation, airway obstruction, inflammation and progressive tissue destruction [1].

The population of patients with bronchiectasis is extremely heterogeneous, representing a group of disorders with a wide range of causes, and varying clinical, radiological and microbiological features [2]. There are no licenced therapies for bronchiectasis and limited evidence even for widely used treatments, such as physiotherapy and long-term macrolide treatment. Recently, a therapeutic approach based on the severity of the disease has been suggested [3]. A step towards a more individualised management of bronchiectasis patients would be the identification of distinct clinical phenotypes using a multidimensional approach that includes parameters available in daily clinical practice.

The majority of therapeutic approaches in bronchiectasis are aimed at the management of chronic bacterial infections, with short- and long-term antibiotic courses [3]. It is not known, however, if those patients with chronic bacterial colonisation or colonisation with Pseudomonas aeruginosa present different clinical characteristics to those patients without. The clinical heterogeneity that characterises patients with bronchiectasis might reflect different pathophysiological mechanisms, which could be considered potential targets for therapeutic interventions. Cluster analysis or unsupervised machine learning techniques have been used successfully to identify biological and clinical subgroups or "phenotypes" in other respiratory disorders, such as asthma and chronic obstructive pulmonary disease (COPD) [4].

In light of the heterogeneity that characterises patients with bronchiectasis, and the need for better directing current management and targeting future treatments, we hypothesised that among these patients there would be discrete groups of subjects with different clinical and biological characteristics.

\section{Materials and methods Study population}

This was a secondary analysis of five databases of prospectively enrolled outpatients with bronchiectasis referred to the bronchiectasis clinics of university teaching hospitals in Monza (Italy), Dundee (UK), Leuven (Belgium), Athens (Greece) and Galway (Ireland). Consecutive patients aged $\geqslant 18$ years with a diagnosis of bronchiectasis on high-resolution computed tomography (HRCT) scan in the stable state were enrolled. Patients with cystic fibrosis or traction bronchiectasis due to pulmonary fibrosis were excluded. A further exclusion criterion for the Athens cohort was the use of antibiotics in the prior 4 weeks and for the Leuven cohort was the presence of active cancer. Collection of selected variables was approved at each individual centre by the local ethical committee or institutional review board.

\section{Data collection}

At the time of clinical assessment, all patients underwent the same comprehensive diagnostic work-up in each site as suggested by the 2010 British Thoracic Society (BTS) guidelines [1]. Demographics, comorbidities, disease severity, aetiology of bronchiectasis, respiratory symptoms, sputum evaluation, radiological, functional and laboratory findings in the stable state, quality of life, long-term treatments, and outcomes (including exacerbations, hospitalisations and mortality) during a 3-year follow-up were uniformly recorded in each database.

The Charlson Comorbidity Index (CCI) was used to assess comorbidities; this is a sum score of 19 weighted diseases, with higher scores denoting increasing burden of comorbidities [5]. The severity of bronchiectasis was evaluated according to both the Bronchiectasis Severity Index (BSI) and FACED score (evaluating forced expiratory volume in $1 \mathrm{~s}$, age, chronic infection with Pseudomonas, radiological extension and dyspnoea) [6,7]. Radiological severity of bronchiectasis was assessed using a modified Reiff score, which rates the number of involved lobes (with the lingula considered to be a separate lobe) and the degree of dilatation (range 1-18) [8]. Patients completed the St George's Respiratory Questionnaire (SGRQ) as a measure of quality of life [9]. All bacteriology was performed on spontaneous sputum samples as described previously [10]. Murray-Washington criteria for sputum quality was used in all cases, with all samples having less than 10 squamous cells and more than 25 leukocytes per low-power microscope field. Chronic infection was defined by the isolation of potentially pathogenic bacteria in sputum culture on two or more occasions, at least 3 months apart over a 1-year period [11]. The predominant pathogen was the organism grown most frequently over the study period. Patients who were unable to provide sputum samples due to absence of a productive cough were classified as not having chronic infection for the purposes of analysis. 


\section{Study outcomes}

\section{Exacerbations}

An exacerbation of bronchiectasis was defined as a clinical diagnosis of exacerbation for which antibiotics were prescribed in the presence of at least one (and usually more than one) of the following symptoms: increasing cough, increasing sputum volume, worsening sputum purulence, worsening dyspnoea, increased fatigue/malaise, fever and haemoptysis [1].

\section{Hospitalisation for severe exacerbations}

Severe exacerbations were defined according to the BTS guidelines as unscheduled hospitalisations or emergency department visits for severe bronchiectasis exacerbations or complications, and were recorded from patient histories and verified using administrative databases [1].

\section{Mortality}

All-cause mortality for up to 3 years was evaluated.

\section{Statistical analysis}

An electronic form was used to collect epidemiological, demographic, clinical and follow-up variables in the clinical centres participating in the project. Qualitative and quantitative variables were summarised using relative frequencies (percentages) and medians (interquartile ranges (IQRs)), when appropriate. Chi-squared and Kruskal-Wallis tests were performed to evaluate qualitative and quantitative variables, respectively. A p-value of $<0.05$ was used to consider a difference statistically significant. A Spearman correlation analysis was performed between all the collected variables; $\rho>0.3$ was used to select variables to be included in the principal component analysis (PCA). A cut-off eigenvalue of 0.7 was adopted to choose the components and a factor loading of 0.4 was considered to identify the most important variables to be selected in the cluster analysis [12]. After hierarchical analysis, a dendrogram was prepared to visually assess the distribution of the clusters related to the recruited cohort. The number of clusters was decided on the basis of a Gower dissimilarity value of 20. A descriptive and an inferential analysis of the collected variables in between the selected clusters was carried out to detect any statistical differences [13]. All the statistical analyses were performed with Stata version 13 statistical software (StataCorp, College Station, TX, USA).

\section{Validation cohort}

In order to validate the primary cluster analysis, we recruited an independent population of patients with HRCT-confirmed bronchiectasis at Ninewells Hospital, Dundee, UK, recruited during 2014. We hypothesised that the clinical clusters identified in the primary study would show differences in neutrophil-mediated inflammation. The validation study was conducted as a case-control study, with a consecutive cohort of 30 patients recruited in each of the four identified phenotype arms (identified by their primary clustering characteristic) (see online supplementary material). Spontaneous sputum samples were obtained and ultracentrifuged at $50000 \times \mathrm{g}$ for $90 \mathrm{~min}$ to obtain supernatant for inflammatory marker measurement as described previously [10]. Clinical evaluation was conducted as described above.

\section{Results}

\section{Study population}

A total of 1145 patients were enrolled in the five centres (median (IQR) age 66 (56-74) years; 40\% males): 286 patients in Dundee, 280 in Galway, 230 in Monza, 190 in Leuven and 159 in Athens. Demographics, clinical, functional and radiological status, microbiology, severity of the disease, and long-term antibiotic treatment of the entire study population are presented in table 1. A detailed overview of the five cohorts is reported in online supplementary table S1. PCA and cluster analysis, which are reported in the online supplementary material, allowed the identification of four clusters.

\section{Cluster characteristics}

The clinical features of the four clusters are shown in table 2. 13 patients were allocated in none of the four clusters because of missing data and excluded from further analysis. The study population was almost equally distributed among the four clusters. Age, sex, body mass index and CCI were not significantly different among the four clusters. However, the presence and type of chronic infection were the key factors defining two of the four clusters.

Cluster 1 included $16 \%(\mathrm{n}=179)$ of the patients and all of them had chronic infection with P. aeruginosa. In comparison with all the other clusters, the patients belonging to this cluster presented the most severe disease, showing the worst radiological and the highest inflammatory patterns, the lowest functional status, 


\section{TABLE 1 Patient characteristics of the entire study population}

\section{Study cohort}

Demographics and comorbidities

Age years

Male

$\mathrm{BMI} \mathrm{kg} \cdot \mathrm{m}^{-2}$

Smoker/ex-smoker

$\mathrm{CCl}>1$

\section{Aetiology of bronchiectasis}

Idiopathic

Post-infective

373 (34)

COPD

$290(26)$

$120(11)$

Connective tissue disease

$89(8)$

Immunodeficiency

$56(5)$

$54(4.9)$

ABPA

$35(3.2)$

Inflammatory bowel disease

$24(2.2)$

Ciliary dysfunction

$20(1.8)$

Aspiration

$14(1.3)$

$\alpha_{1}$-antitrypsin deficiency

$10(0.9)$

Congenital

$5(0.5)$

Other

$20(1.8)$

Disease severity

BSI score

FACED score

Radiological status

Reiff score

\section{Clinical status}

Daily cough

$899(79)$

Daily sputum

$744(65)$

Prior history of haemoptysis

$206(18)$

MRC breathlessness scale

$2(1-3)$

Long-term oxygen therapy

$86(7.5)$

Exacerbations in the previous year

$2(1-3)$

At least one hospitalisation in the previous year

\section{Functional status}

FEV $1 \%$ predicted

Microbiology

Chronic infection with at least one pathogen ${ }^{\#}$

Haemophilus influenzae

$185(16)$

Pseudomonas aeruginosa

Meticillin-sensitive Staphylococcus aureus

$61(5.3)$

Moraxella catarrhalis

$51(4.5)$

Streptococcus pneumoniae

$47(4.1)$

Enterobacteriaceae

$29(2.5)$

Meticillin-resistant Staphylococcus aureus

$21(1.8)$

Escherichia coli

Klebsiella pneumoniae

$4(1.2)$

$13(1.1)$

Nontuberculous mycobacteria

$8(0.7)$

Other

Laboratory findings

C-reactive protein $\mathrm{mg} \cdot \mathrm{L}^{-1}$

$6(4-8)$

Long-term antibiotic treatment

Either macrolide or inhaled antibiotics

388 (34)

Macrolide

Inhaled antibiotic treatment

$90(7.9)$

Both macrolide and inhaled antibiotics

Data are presented as $\mathrm{n}$, median (interquartile range) or $\mathrm{n}(\%)$. BMI: body mass index; CCl: Charlson Comorbidity Index; COPD: chronic obstructive pulmonary disease; ABPA: allergic bronchopulmonary aspergillosis; BSI: Bronchiectasis Severity Index; MRC: Medical Research Council; FEV1: forced expiratory volume in $1 \mathrm{~s} . ~ "$ : 150 patients had more than one pathogen as the cause of chronic infection; among those, 56 patients had Pseudomonas aeruginosa plus another bacterial species. 
TABLE 2 Baseline characteristics in the four clusters

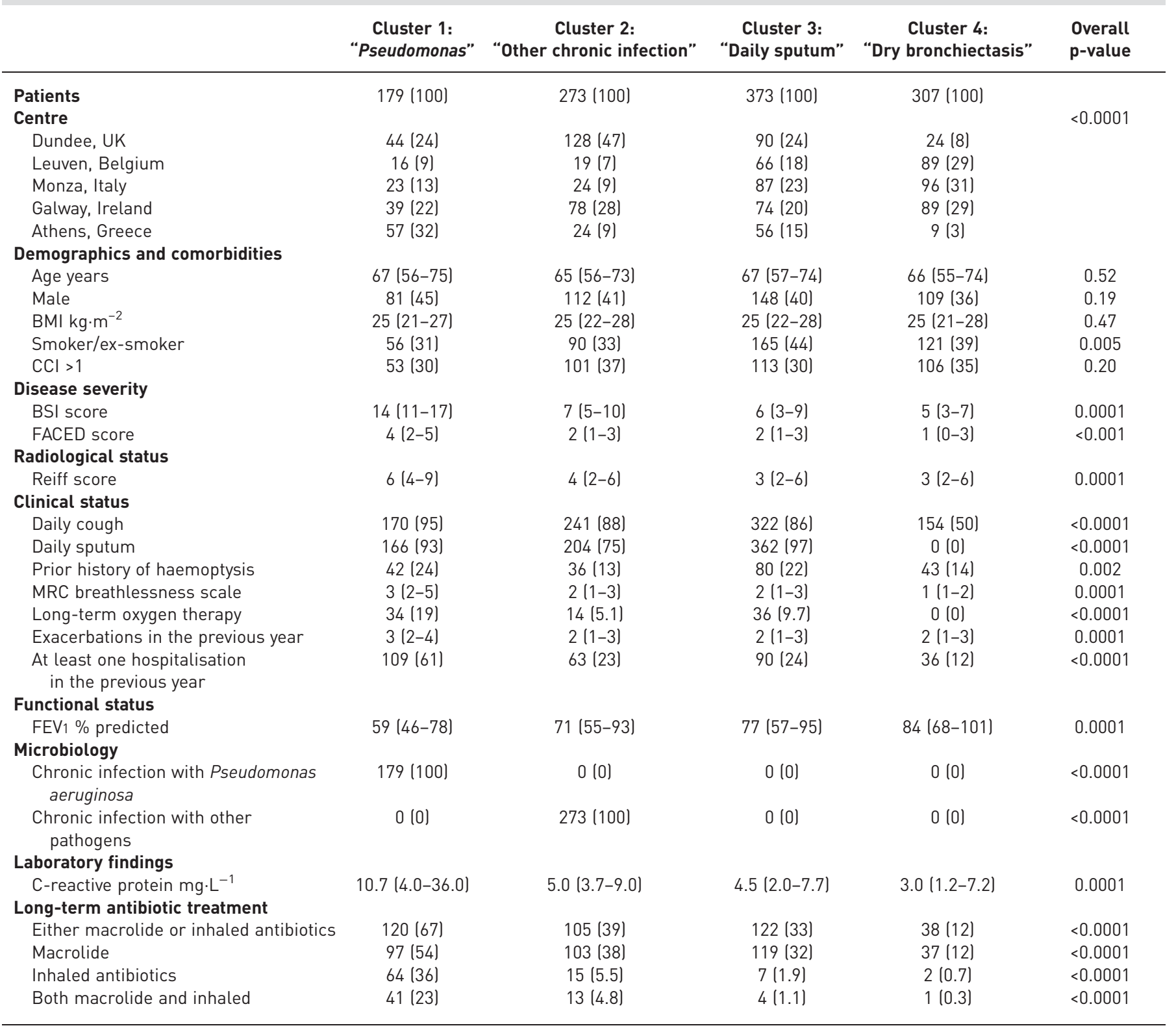

Data are presented as $\mathrm{n}(\%)$ or median (interquartile range), unless otherwise stated. BMI: body mass index; CCI: Charlson Comorbidity Index; BSI: Bronchiectasis Severity Index; MRC: Medical Research Council; FEV1: forced expiratory volume in $1 \mathrm{~s}$.

the highest number of exacerbations and hospitalisations, and the worst quality of life at baseline. Based on these characteristics, Cluster 1 was labelled as "Pseudomonas".

There were two intermediate clusters in terms of disease severity (i.e. Clusters 2 and 3), including patients with moderate levels of systemic inflammation and functional status, of whom $20 \%$ experienced at least one hospitalisation per year.

Cluster 2 included 24\% $(n=273)$ of the study population and was characterised by the presence of chronic infection with pathogens other than P. aeruginosa. Accordingly, this cluster was labelled as "Other chronic infection".

Cluster 3 was the largest cluster, including 33\% (n=373) of the study population. No patients within this cluster had chronic infection, but almost all of them had daily sputum and a slightly higher proportion were smokers or ex-smokers. Accordingly, this cluster was labelled as "Daily sputum". 
Cluster 4 included $27 \%(n=307)$ of the patients. These patients were generally of lower severity, showing the lowest level of inflammatory biomarkers, the least severe radiological and less functional impairment. None of these patients had chronic infection and none had daily sputum. Accordingly, this cluster was labelled as "Dry bronchiectasis".

The distribution of the four clusters within each study centres is given in online supplementary table S2.

The aetiology of bronchiectasis was identified in $66 \%(n=756)$ of the patients. Excluding idiopathic bronchiectasis, the first three most commonly defined aetiologies were post-infective $(\mathrm{n}=290(26 \%))$, COPD-related $(n=120(11 \%))$ and connective tissue disease-related $(n=89(8 \%))$ (table 1). A detailed distribution of different aetiologies among the four clusters is given in table 3 . No clinically and statistically significant differences in terms of bronchiectasis aetiology were detected among the four clusters, apart from post-infective and COPD-related bronchiectasis.

A significant difference in terms of long-term antibiotic treatment, including macrolides and inhaled antibiotics, was detected among the four clusters (table 2). More than $50 \%$ of the patients in the "Pseudomonas", and one-third of the patients in the "Other chronic infection" and "Daily sputum" clusters were exposed to a macrolide. More than one-third of the patients in the "Pseudomonas" cluster were on inhaled antibiotic treatment, while few patients received this treatment in the other clusters.

\section{Quality of life and clinical outcomes during follow-up}

Quality of life was evaluated in 389 (34\%) patients, showing a median (IQR) SGRQ value of 39 (26-58). Significant differences in median values of SGRQ were detected across the four clusters, with the worst quality of life for patients in the "Pseudomonas" cluster (table 4).

Significant differences in terms of both exacerbations and hospitalisations due to exacerbations were detected along the four clusters during the 1-year follow-up, with the higher rates detected in the "Pseudomonas" and "Other chronic infection" clusters. At 1-year follow-up, no significant differences in mortality were detected among the different clusters; however, the "Pseudomonas" cluster showed a significant higher mortality rate during the 3-year follow-up period (table 4).

\section{Biological and clinical validity of the clusters}

A total of 120 patients were included in a case-control study, using the major characteristics, i.e. presence of $P$. aeruginosa, chronic colonisation with other pathogens and daily sputum production without colonisation, or absence of these three characteristics (table 5). Patients in the "Pseudomonas", "Other chronic infection" and "Daily sputum" clusters were recruited consecutively. As sputum production was less frequent in the "Dry bronchiectasis" cluster, 66 patients were recruited in order to obtain 30 patients with sputum samples available for analysis. Higher levels of free neutrophil elastase activity, myeloperoxidase (MPO) activity and interleukin (IL)-1 $\beta$ were found in the "Pseudomonas" and "Other chronic infection" clusters, while there were no differences in other cytokines (figure 1).

TABLE 3 Aetiology of bronchiectasis in the four clusters

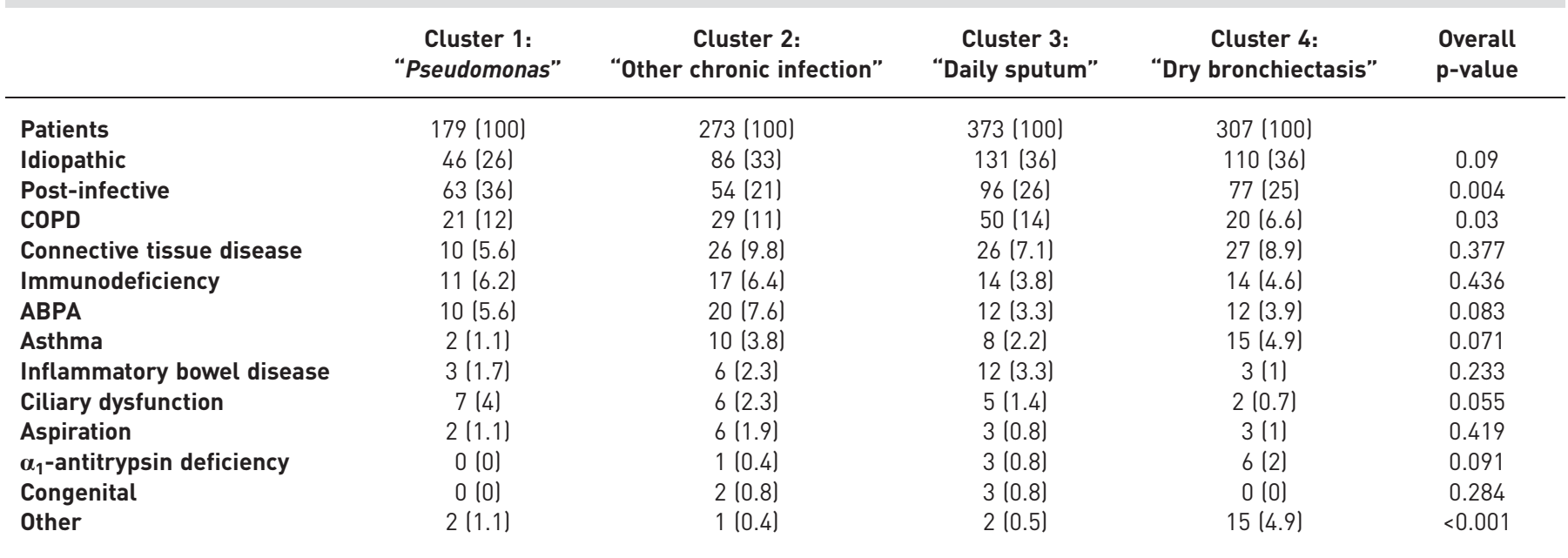

Data are presented as $\mathrm{n}(\%)$, unless otherwise stated. COPD: chronic obstructive pulmonary disease; ABPA; allergic bronchopulmonary aspergillosis. 
TABLE 4 Quality of life and longitudinal outcomes in the four clusters

\begin{tabular}{|c|c|c|c|c|c|}
\hline & $\begin{array}{c}\text { Cluster 1: } \\
\text { "Pseudomonas" }\end{array}$ & $\begin{array}{c}\text { Cluster 2: } \\
\text { "Other chronic infection” }\end{array}$ & $\begin{array}{c}\text { Cluster 3: } \\
\text { “Daily sputum" }\end{array}$ & $\begin{array}{c}\text { Cluster 4: } \\
\text { “Dry bronchiectasis" }\end{array}$ & $\begin{array}{l}\text { Overall } \\
\text { p-value }\end{array}$ \\
\hline Patients & $179(100)$ & $273(100)$ & $373(100)$ & $307(100)$ & \\
\hline SGRQ & $58(34-72)$ & $43(27-61)$ & $39(27-55)$ & $29(12-40)$ & $<0.001$ \\
\hline \multicolumn{6}{|l|}{ Outcomes } \\
\hline Exacerbations during 1-year follow-up & $2(1-3)$ & $2(1-2)$ & $1(0-2)$ & $1(0-2)$ & 0.0001 \\
\hline Mortality during 1-year follow-up & $9(5.1)$ & $4(1.5)$ & $13(3.6)$ & $14(4.9)$ & 0.12 \\
\hline Mortality during 3-year follow-up & $26(17)$ & $19(7.6)$ & $24(8.2)$ & $23(11)$ & 0.02 \\
\hline
\end{tabular}

Data are presented as $\mathrm{n}(\%)$ or median (interquartile range), unless otherwise stated. SGRQ: St George's Respiratory Questionnaire.

\section{Discussion}

The major finding of our study is the evidence of microbiology, including chronic infections with $P$. aeruginosa or other pathogens, and the daily presence of sputum production as the major drivers to classify patients with bronchiectasis in four discrete groups. We further demonstrated that these clusters represent clinical phenotypes because they exhibit differences in terms of not only inflammatory biomarkers on sputum, but also quality of life and long-term clinical outcomes.

PCA and cluster analysis have been successfully used in recent years to classify patients with asthma and COPD in discrete groups according to similar combinations of disease characteristics [14-23]. No previous studies have used PCA and cluster analysis to identify clinical phenotypes in bronchiectasis. Previous

TABLE 5 Patient characteristics and quality of life in the validation cohort according to the four clusters

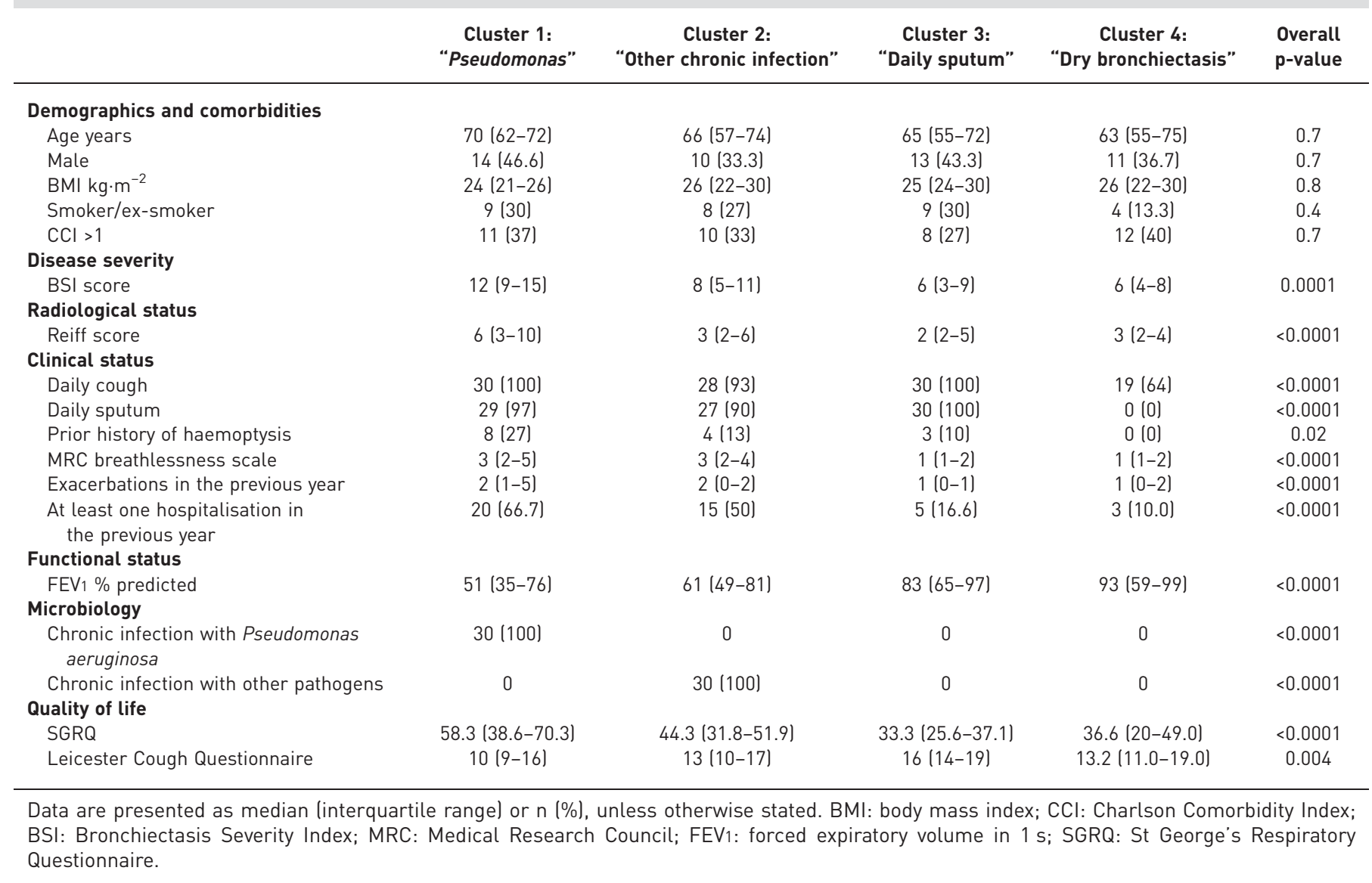



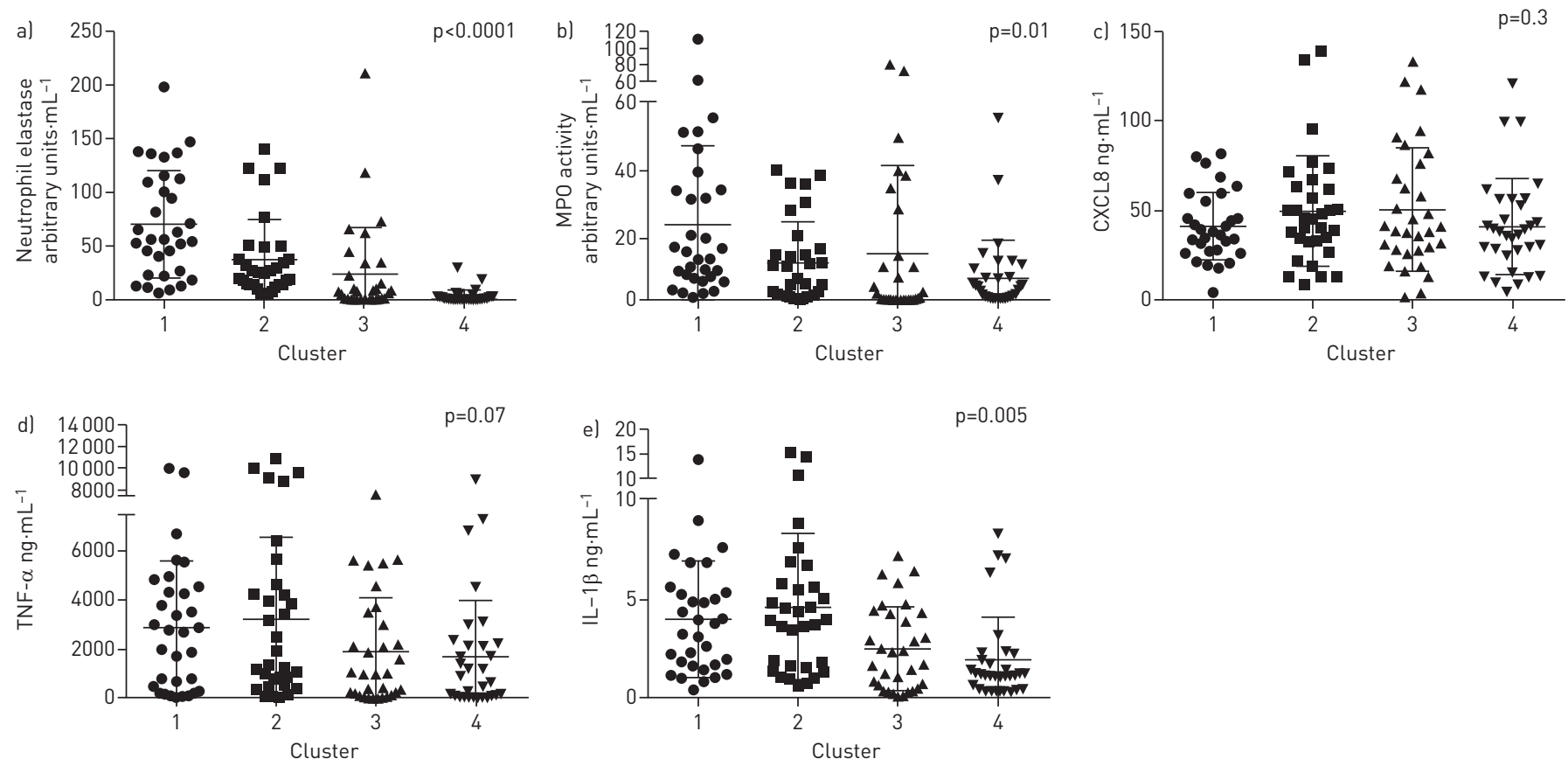

FIGURE 1 Sputum markers of inflammation among different clusters in the validation cohort: a) neutrophil elastase, b) myeloperoxidase (MPO), c) chemokine CXCL8 (interleukin (IL)-8)), d) tumour necrosis factor (TNF)- $\alpha$ and e) IL-1 $\beta$. Bars indicate mean \pm SD. Cluster 1: "Pseudomonas"; cluster 2: "Other chronic infection"; cluster 3: "Daily sputum"; cluster 4: "Dry bronchiectasis".

literature defined disease severity through the identification of predictors of mortality and hospital admissions using multivariable analysis [6, 7]. Although scores such as the BSI and FACED scores might recognise which patient is globally severe, they do not help physicians in identifying which aspects of patient management should be addressed in daily clinical practice. The present analysis, which does not include the derivation cohort of the BSI paper [6], sought clusters that were not necessarily related to outcomes, but rather patient characteristics providing important new information beyond the available scoring systems.

The presence of chronic infection with Pseudomonas seems to define by itself a specific clinical phenotype of patients with bronchiectasis who share a more relevant inflammatory status, a more severe disease, worse clinical, functional and radiological characteristics, and worse quality of life and long-term outcomes, in line with previous literature [24]. Two-thirds of the patients in the "Pseudomonas" cluster were on long-term antibiotic treatment, with half of the patients receiving a macrolide. From a clinical perspective, our findings highlight the importance of sputum surveillance for all patients with bronchiectasis; from a research perspective, they advocate for better evidence on the effectiveness and safety of eradication or long-term suppressive therapy for $P$. aeruginosa infection. Furthermore, the significance and impact of intermittent infection with P. aeruginosa (as well as other pathogens) requires evaluation in future studies. Finally, the "Pseudomonas" cluster characterised only $16 \%$ of the bronchiectasis patients in our cohort, prompting the need to better evaluate characteristics of other phenotypes in order to individualise and optimise management.

Patients with chronic infections other than $P$. aeruginosa constitute a distinct clinical phenotype accounting for $24 \%$ of the patients with bronchiectasis. A recent study demonstrated worse outcomes for patients with a chronic infection due to pathogens other than $P$. aeruginosa compared with patients without a chronic infection, but to a lesser extent than those infected with P. aeruginosa [6]. Our findings related to the "Other chronic infection" cluster, showing intermediate rates of exacerbations, hospitalisations and mortality in between the "Pseudomonas" and the "Dry bronchiectasis" clusters, confirmed these data. More than one-third of the patients in the "Other chronic infection" cluster received long-term antibiotic treatment, especially macrolides, with only $5.5 \%$ exposed to inhaled antibiotics. There is a paucity of data in the literature concerning the evaluation of inhaled antibiotics specifically developed for chronic infections with bacteria other than $P$. aeruginosa, although the percentage of these patients in the bronchiectasis population is not negligible and they do suffer from adverse outcomes.

The largest clinical phenotype in our cohort, i.e. the "Daily sputum" cluster, was composed of patients with daily sputum production and without chronic infections. These patients had similar levels of disease 
severity and systemic inflammation, with a similar history of exacerbations, clinical outcomes and chronic antibiotic use in comparison with patients belonging to the "Other chronic infection" cluster. These patients may require interventions to enhance sputum clearance, smoking cessation advice if they are active smokers and sputum screening to identify the presence of chronic infection. It would be conceivable that, although we were not able to identify a chronic infection, with standard methods, the use of new techniques, such as $16 \mathrm{~S}$ rRNA gene pyrosequencing, could allow the microbiological classification of those patients, broadening potential therapeutic options [25].

Patients in the "Dry bronchiectasis" cluster showed the lowest systemic inflammation level, disease severity and clinical impact of bronchiectasis, with the best functional status and without any chronic infections. Very few data have been reported in the literature regarding mild or early-stage bronchiectasis, and certainly further clinical and translational research is needed to identify early therapeutic targets in order to prevent disease progression.

Notably, the distribution of single clusters was significantly different among the five study centres. It should be acknowledged that all of them are tertiary-care bronchiectasis clinics and this difference could be mainly attributed to not only to different disease characteristics that might exist across different European regions, but also to a referral bias due to different healthcare systems.

When we validated our findings in an independent cohort of patients with bronchiectasis, we found that this classification based on the predominant feature of each cluster (chronic infection with Pseudomonas or other bacteria, daily sputum or the absence of daily sputum) is clinically valid with clear differences in lung function, symptoms and disease severity between groups consistent with the primary analysis. In addition, we found higher levels of neutrophil elastase, MPO and IL-1 $\beta$ in the "Pseudomonas" and "Other chronic infection" clusters, while there were no differences in other cytokines. This is very much consistent with previous published data showing higher levels of neutrophil inflammatory markers in patients with bacterial colonisation and higher bacterial loads [10]. This previous literature, together with the present findings, confirms that the bacterial colonisation status is the most useful clinical parameter to subclassify patients.

This study is the first to apply cluster analysis principles for the identification of clinical phenotypes in bronchiectasis. It represents one of the largest cohorts of patients with bronchiectasis published so far in the literature, including patients from five European countries with a 3-year follow-up. The sample size and the multicentre design increase the importance of our findings. One of the strengths of the current analysis is the identification of different phenotypes in bronchiectasis through variables that are usually collected during daily clinical practice. Finally, the presence of differences in sputum biomarkers and quality of life in a validation cohort strengthens the validity of these phenotypes.

One of the limitations of the present study is that, although we merged multiple cohorts obtained in different settings across Europe to ensure representation of different subgroups of patients, all centres were tertiary-care centres enrolling a selected population of patients. Further studies should also include cohorts of in/outpatients recruited in both secondary- and primary-care settings, and also collect other important comorbidities not assessed by the CCI, such as depression or anxiety. Furthermore, we did not collect the cause and the exact date of death in all the cohorts, and thus we were not able to perform a survival analysis on our cohort.

Cluster analysis, using clinical, microbiological, functional and radiological variables, identified four clinical phenotypes easily detected according to the presence of chronic infection with $P$. aeruginosa or other pathogens and daily sputum. Patients belonging to these clinical phenotypes show distinctly different patterns of sputum biomarkers, quality of life and outcomes. Identification of clinical phenotypes showing similar biological profiles and prognosis could favour a focused treatment as well as test interventions in further randomised controlled trials designed to alter the natural history of the disease.

\section{References}

1 Pasteur MC, Bilton D, Hill AT, et al. British Thoracic Society guideline for non-CF bronchiectasis. Thorax 2010; 65: Suppl. 1, i1-i58.

Barker AF. Bronchiectasis. N Engl J Med 2002; 346: 1383-1393.

Chalmers JD, Aliberti S, Blasi F. Management of bronchiectasis in adults. Eur Respir J 2015; 45: 1446-1462.

Han MK, Agusti A, Calverley PM, et al. Chronic obstructive pulmonary disease phenotypes: the future of COPD. Am J Respir Crit Care Med 2010; 182: 598-604.

5 Charlson ME, Pompei P, Ales KL, et al. A new method of classifying prognostic comorbidity in longitudinal studies: development and validation. J Chronic Dis 1987; 40: 373-383.

6 Chalmers JD, Goeminne P, Aliberti S, et al. The Bronchiectasis Severity Index. An international derivation and validation study. Am J Respir Crit Care Med 2014; 189: 576-585.

7 Martínez-García MÁ, de Gracia J, Vendrell Relat M, et al. Multidimensional approach to non-cystic fibrosis bronchiectasis: the FACED score. Eur Respir J 2014; 43: 1357-1367. 
Reiff DB, Wells AU, Carr DH, et al. CT findings in bronchiectasis: limited value in distinguishing between idiopathic and specific types. AJR Am J Roentgenol 1995; 165: 261-267.

9 Wilson CB, Jones PW, O'Leary CJ, et al. Validation of the St. George's Respiratory Questionnaire in bronchiectasis. Am J Respir Crit Care Med 1997; 156: 536-541.

10 Chalmers JD, Smith MP, McHugh BJ, et al. Short- and long-term antibiotic treatment reduces airway and systemic inflammation in non-cystic fibrosis bronchiectasis. Am J Respir Crit Care Med 2012; 186: 657-665.

11 Pasteur MC, Helliwell SM, Houghton SJ, et al. An investigation into causative factors in patients with bronchiectasis. Am J Respir Crit Care Med 2000; 162: 1277-1284.

12 Jolliffe IT. Discarding variables in a principal component analysis. II: real data. Appl Stat 1973; 22: 21-31.

13 Bartholomew DJ, Steele F, Galbraith J, et al. Analysis of Multivariate Social Science Data. 2nd Edn. London, Chapman \& Hall/CRC, 2008.

14 Haldar P, Pavord ID, Shaw DE, et al. Cluster analysis and clinical asthma phenotypes. Am J Respir Crit Care Med 2008; 178: 218-224

15 Everitt B, Landau S, Leese M, et al. Cluster Analysis. 5th Edn. Hoboken, Wiley, 2011.

16 Burgel PR, Paillasseur JL, Caillaud D, et al. Clinical COPD phenotypes: a novel approach using principal component and cluster analyses. Eur Respir J 2010; 36: 531-539.

17 Vanfleteren LE, Spruit MA, Groenen M, et al. Clusters of comorbidities based on validated objective measurements and systemic inflammation in patients with chronic obstructive pulmonary disease. Am J Respir Crit Care Med 2013; 187: 728-735.

18 Burgel PR, Roche N, Paillasseur JL, et al. Clinical COPD phenotypes identified by cluster analysis: validation with mortality. Eur Respir J 2012; 40: 495-496.

19 Garcia-Aymerich J, Gómez FP, Benet M, et al. Identification and prospective validation of clinically relevant chronic obstructive pulmonary disease (COPD) subtypes. Thorax 2011; 66: 430-437.

20 Cho MH, Washko GR, Hoffmann TJ, et al. Cluster analysis in severe emphysema subjects using phenotype and genotype data: an exploratory investigation. Respir Res 2010; 11: 30.

21 Burgel PR, Paillasseur JL, Peene B, et al. Two distinct chronic obstructive pulmonary disease (COPD) phenotypes are associated with high risk of mortality. PLoS One 2012; 7: e51048.

22 Castaldi PJ, Dy J, Ross J, et al. Cluster analysis in the COPDGene study identifies subtypes of smokers with distinct patterns of airway disease and emphysema. Thorax 2014; 69: 415-422.

23 Rennard SI, Locantore N, Delafont B, et al. Identification of five chronic obstructive pulmonary disease subgroups with different prognoses in the ECLIPSE cohort using cluster analysis. Ann Am Thorac Soc 2015; 12: 303-312.

24 Finch S, McDonnell MJ, Abo-Leyah H, et al. A comprehensive analysis of the impact of Pseudomonas aeruginosa colonisation on prognosis in adult bronchiectasis. Ann Am Thorac Soc 2015; 12: 1602-1611.

25 Tunney MM, Einarsson GG, Wei L, et al. Lung microbiota and bacterial abundance in patients with bronchiectasis when clinically stable and during exacerbation. Am J Respir Crit Care Med 2013; 187: 1118-1126. 